(C) The Authors 2019. This is an Open Access article, distributed under the terms of the Creative Commons Attribution licence (http:// creativecommons.org/licenses/by/4.0/), which permits unrestricted re-use, distribution, and reproduction in any medium, provided the original work is properly cited.

\title{
New approach to simplifying and optimising acute malnutrition treatment in children aged 6-59 months: the OptiMA single-arm proof-of-concept trial in Burkina Faso
}

\author{
Maguy Daures ${ }^{1 * \dagger}$, Kevin Phelan ${ }^{2} \dagger$, Mariama Issoufou ${ }^{3}$, Séni Kouanda ${ }^{4}$, Ousmane Sawadogo \\ Kader Issaley ${ }^{6}$, Cecile Cazes ${ }^{1}$, Benjamin Séri ${ }^{7}$, Bertine Ouaro ${ }^{8}$, Bruno Akpakpo ${ }^{3}$, Vincent Mendiboure ${ }^{6}$, \\ Susan Shepherd ${ }^{6} \dagger$ and Renaud Becquet ${ }^{1} \dagger$ \\ ${ }^{1}$ University of Bordeaux, Inserm, Bordeaux Population Health Research Center, team IDLIC, UMR 1219, Bordeaux, France \\ ${ }^{2}$ The Alliance for International Medical Action (ALIMA), Paris, France \\ ${ }^{3}$ The Alliance for International Medical Action (ALIMA), Yako, Burkina Faso \\ ${ }^{4}$ Département biomédical et santé publique, Institut de Recherche en Sciences de la Sante, Ouagadougou, Burkina Faso \\ ${ }^{5}$ Association KEOOGO, Ouagadougou, Burkina Faso \\ ${ }^{6}$ The Alliance for International Medical Action (ALIMA), Dakar, Senegal \\ ${ }^{7}$ PACCI research programme, University Hospital of Treichville, Abidjan, Ivory Coast \\ ${ }^{8}$ Ministry of Health, Ouagadougou, Burkina Faso
}

(Submitted 1 July 2019 - Final revision received 21 October 2019 - Accepted 3 December 2019 - First published online 10 December 2019)

\section{Abstract}

The Optimising treatment for acute MAlnutrition (OptiMA) strategy trains mothers to use mid upper arm circumference (MUAC) bracelets for screening and targets treatment to children with MUAC $<125 \mathrm{~mm}$ or oedema with one therapeutic food at a gradually reduced dose. This study seeks to determine whether OptiMA conforms to SPHERE standards (recovery rate $>75 \%$ ). A single-arm proof-of-concept trial was conducted in 2017 in Yako district, Burkina Faso including children aged 6-59 months in outpatient health centres with MUAC < 125 mm or oedema. Outcomes were stratified by MUAC category at admission. Multivariate survival analysis was carried out to identify variables predictive of recovery. Among 4958 children included, 824 (16.6\%) were admitted with MUAC < 115 mm or oedema, 1070 (21.6\%) with MUAC 115-119 mm and $3064(61.8 \%)$ with MUAC 120-124 mm. The new dosage was correctly implemented at all visits for $75.9 \%$ of children. Global recovery was $86.3(95 \%$ CI $85 \cdot 4,87 \cdot 2) \%$ and 70.5 (95\% CI 67.5, 73.5) \% for children admitted with MUAC < $115 \mathrm{~mm}$ or oedema. Average therapeutic food consumption was 60.8 sachets per child treated. Recovery was positively associated with mothers trained to use MUAC prior to child's admission (adjusted hazard ratio 1.09; $95 \%$ CI 1.01, 1.19). OptiMA was successfully implemented at the scale of an entire district under 'real-life' conditions. Programme outcomes exceeded SPHERE standards, but further study is needed to determine if increasing therapeutic food dosages for the most severely malnourished will improve recovery.

Key words: Acute malnutrition: Children: West Africa: Mid upper arm circumference

Acute malnutrition is a major public health problem, affecting 49.5 million children worldwide each year and contributing to nearly half of all annual childhood deaths ${ }^{(1,2)}$. One quarter of all acutely malnourished children are in Africa with the largest number, $5 \cdot 1$ million, in West Africa ${ }^{(2)}$. In this region, the burden of acute malnutrition overlaps with the highest rates of child mortality $^{(2)}$. Progress in reducing acute malnutrition needs to accelerate in the region if it is to meet WHO Global Nutrition Targets by $2025^{(3)}$.

Although acute malnutrition is a continuum condition, it is arbitrarily divided into moderate (MAM) and severe (SAM) categories defined by mid upper arm circumference (MUAC) or weight-for-height $Z$-score (WHZ). MAM (MUAC 115-124 mm or WHZ between -2 and -3 ) and SAM (MUAC $<115 \mathrm{~mm}$ or

Abbreviations: AF, attributable fraction; MAM, moderate acute malnutrition; MUAC, mid upper arm circumference; OptiMA, Optimising treatment for acute Malnutrition; RUTF, ready-to-use therapeutic food; SAM, severe acute malnutrition; WHZ, weight-for-height $Z$-score.

* Corresponding author: Maguy Daures, email maguy.daures@coral.alimango

$\dagger$ These authors contributed equally to this work. 
WHZ $<-3$ or oedema) are managed separately, with programmes overseen by different UN agencies, and using different protocols and products (ready-to-use supplementary food or fortified-blended flours for MAM and ready-to-use therapeutic food (RUTF) for SAM). Such separation complicates delivery of care, contributes to low coverage and creates confusion among caregivers ${ }^{(4)}$.

Confusion is further exacerbated by a complicated case definition using MUAC and WHZ to determine programme eligibility, even though WHZ does not offer a clear advantage over MUAC for identifying children at near-term risk of death ${ }^{(5)}$. MUAC-only programming is expanding as evidence accumulates that weight gain and MUAC gain track each other and that the trajectory of weight and MUAC gain is maximal during the first 2-3 weeks of treatment ${ }^{(6-8)}$. MUAC is therefore becoming a stand-alone practical tool for all phases of nutrition programming: screening, admission, monitoring recovery and determining discharge.

In addition to the tangle of agencies and case definitions, SAM and MAM programmes are chronically underfunded with only $25 \%$ of SAM cases treated globally in 2016 and $16 \%$ of MAM cases reached by the World Food Program in $2017^{(9,10)}$. An optimised allocation of resources is therefore needed. A programme integrating SAM and MAM treatment in a single MUAC-based protocol using only RUTF showed the cost of RUTF for integrated treatment to be less than half that for standard SAM management ${ }^{(11)}$. Other studies found that identification and treatment of children earlier in the wasting process led to fewer hospitalisations and that inpatient care was shown to be twice as costly as outpatient SAM management ${ }^{(12,13)}$. Furthermore, the treatment of MAM has been shown to be cost-effective in reducing mortality risk by more than $10 \%{ }^{(14)}$

We piloted a new MUAC-based and oedema approach for treating acute malnutrition in Burkina Faso with a single-arm proof-of-concept trial called Optimising treatment for acute MAlnutrition (OptiMA). OptiMA made three strategic changes to the current Burkina Faso National Nutrition protocol $^{(15)}$ : screening for acute malnutrition was task-shifted to caretakers via community-based training in the use of MUAC bracelets; the case definition for acute malnutrition was modified to MUAC $<125 \mathrm{~mm}$ and/or oedema and only one product was used for treatment (RUTF) at a gradually reduced dose based on a child's weight and MUAC status. We hypothesised that the OptiMA protocol was at least as effective as the national nutrition protocol in terms of children's recovery rate and other standard programme indicators when compared against international SPHERE standards ${ }^{(16)}$.

\section{Methods}

\section{Location, period and study population}

This single-arm proof-of-concept trial was conducted in Burkina Faso's Yako Health District. In 2016, Yako had a general population of approximately 415000 , with the under-five population estimated at 82000 , and was served by fifty-four health centres plus a district hospital. Each health centre was staffed by a nurse who routinely provided acute malnutrition treatment. Prevalence of MAM and SAM by WHZ just prior to implementation of OptiMA was $8.2 \%$ and $1.9 \%$, respectively ${ }^{(17)}$. Health centre staff were trained on the OptiMA protocol in November and December 2016. Inclusions for the study occurred from January through December 2017, and follow-up of study participants ended in March 2018. Children were considered eligible for enrollment if they were aged 6-59 months with a MUAC $<125 \mathrm{~mm}$ or bipedal oedema and presented to any of the fifty-four outpatient clinics in Yako District.

\section{Study protocol}

Screening for acute malnutrition. A mass community-based campaign was conducted from June to August 2016 to train mothers and caretakers throughout Yako District to use MUAC bracelets and check for oedema in order to screen their own children for malnutrition. Following the mass campaign, family MUAC trainings became a routine activity at all fifty-four health centres and continued to the end of the study.

Admission/discharge criteria, therapeutic feeding products and dosage. Table 1 summarises the differences between the current national protocol and OptiMA, which was implemented in all of the Yako's fifty-four health centres for the duration of the study. Children presenting with $M U A C \geq 125 \mathrm{~mm}$ and WHZ $<-3$ were treated in a 'non-MUAC eligible' arm according to the current national protocol.

In contrast to the weight-based RUTF ration in the national SAM programme, which is fixed at $150-200 \mathrm{kcal} / \mathrm{kg}$ per $\mathrm{d}(628-$ $837 \mathrm{~kJ} / \mathrm{kg}$ per d) for the course of treatment, the OptiMA RUTF ration was calibrated to the child's degree of wasting based on the combination of MUAC status and weight. Thus, more nutritional support was given to the most severely malnourished and then gradually reduced as the child's MUAC increased. Children with MUAC $<115 \mathrm{~mm}$ or oedema received $175 \mathrm{kcal} /$ $\mathrm{kg}$ per d (732 kJ/kg per d) of RUTF. Children with MUAC 115-119 mm, either at admission or during the course of treatment, received $125 \mathrm{kcal} / \mathrm{kg}$ per d (523 kJ/kg per d) of RUTF, and children with MUAC $\geq 120 \mathrm{~mm}$ received $75 \mathrm{kcal} / \mathrm{kg}$ per $\mathrm{d}$ $(314 \mathrm{~kJ} / \mathrm{kg}$ per d) of RUTF (with a minimum of one sachet/d) until discharge from the programme.

Systematic medical treatment. All children underwent malaria rapid testing upon admission and at any point during their participation if clinical signs of malaria were detected. All children with a positive malaria rapid diagnostic test were prescribed an artemisinin-combination treatment. Amoxicillin $90 \mathrm{mg} / \mathrm{kg}$ per $\mathrm{d}$ for $7 \mathrm{~d}$ was prescribed for all children with MUAC $<120 \mathrm{~mm}$ or oedema. Albendazole was given to children if they had no deworming in the previous 4 months.

\section{Defaulter follow-up}

Community health workers traced children who were classified as defaulters to their caretakers' homes and completed a 
Table 1. Admissions and discharge criteria, treatment products and calculation of dosage in the Burkina Faso national and Optimising treatment for acute MAlnutrition (OptiMA) protocols*

\begin{tabular}{|c|c|c|c|c|c|}
\hline \multirow[b]{3}{*}{ Admission } & \multicolumn{2}{|c|}{ National protocol } & \multicolumn{3}{|c|}{ OptiMA protocol } \\
\hline & \multirow{2}{*}{$\begin{array}{c}\text { SAM } \\
\text { MUAC }<115 \text { mm or } \\
\text { WHZ }<-3 \text { or oedema }\end{array}$} & \multirow{2}{*}{$\begin{array}{c}\text { MAM } \\
\text { MUAC } \geq 115 \mathrm{~mm} \text { and } \\
\text { MUAC }<125 \mathrm{~mm} \text { or } \\
-3<\mathrm{WHZ}<-2\end{array}$} & \multicolumn{3}{|c|}{ Acute malnutrition } \\
\hline & & & $\begin{array}{l}\text { MUAC }<115 \mathrm{~mm} \\
\text { or oedema }\end{array}$ & $115 \leq \mathrm{MUAC} \leq 120 \mathrm{~mm}$ & $120 \leq \mathrm{MUAC}<125 \mathrm{~mm}$ \\
\hline $\begin{array}{c}\text { Treatment } \\
\text { product }\end{array}$ & $\begin{array}{l}\text { RUTF } 150-200 \\
\mathrm{kcal} / \mathrm{kg} \text { per d }\end{array}$ & $\begin{array}{l}\text { Super cereal plus } 200 \mathrm{~g} / \mathrm{d} \\
\text { or RUSF one } 92 \mathrm{~g} \\
\text { sachet/d }\end{array}$ & $\begin{array}{l}\text { RUTF } 175 \\
\quad \mathrm{kcal} / \mathrm{kg} \text { per d }\end{array}$ & RUTF $125 \mathrm{kcal} / \mathrm{kg}$ per d & RUTF $75 \mathrm{kcal} / \mathrm{kg}$ per d \\
\hline $\begin{array}{l}\text { Calculation } \\
\text { of dosage }\end{array}$ & According to the weight & $\begin{array}{l}\text { Fixed amount, regardless } \\
\text { of weight or MUAC } \\
\text { status }\end{array}$ & According to MUA & tatus and weight & \\
\hline \multirow[t]{3}{*}{$\begin{array}{l}\text { Discharge } \\
\text { criteria }\end{array}$} & \multicolumn{2}{|c|}{$\begin{array}{l}\text { MUAC } \geq 125 \mathrm{~mm} \text { for two consecutive visits or } \\
\text { WHZ } \geq-2 \text { for two consecutive visits }\end{array}$} & \multicolumn{3}{|c|}{ MUAC $\geq 125 \mathrm{~mm}$ for two consecutive weeks } \\
\hline & $\begin{array}{l}\text { No oedema minimum } \\
2 \text { weeks }\end{array}$ & $\begin{array}{l}\text { After recovery from SAM: } \\
\text { discharge after } 3 \text { months } \\
\text { without losing weight }\end{array}$ & \multicolumn{3}{|c|}{ No oedema for minimum 2 weeks } \\
\hline & $\begin{array}{l}\text { Minimum } 4 \text { weeks in } \\
\text { programme }\end{array}$ & & \multicolumn{3}{|c|}{ Minimum 4 weeks in programme } \\
\hline
\end{tabular}

SAM, severe acute malnutrition; MAM, moderate acute malnutrition; MUAC, mid upper arm circumference; WHZ, weight-for-height Z-score; RUTF, ready-to-use therapeutic food; RUSF, ready-to-use supplementary food.

* To convert kcal to kJ, multiply by 4.184.

standardised form recording vital status, MUAC measure and reasons for defaulting.

\section{Primary and secondary outcomes}

The primary outcome was recovery defined as the proportion of children achieving a MUAC $\geq 125 \mathrm{~mm}$ and absence of oedema for two consecutive weeks, in good clinical health, with a minimum programme stay of 4 weeks. Secondary outcomes were RUTF consumption and other standard programme indicators: death (occurring after programme admission and prior to recovery), default (being absent for three consecutive visits), non-response (failure to achieve recovery after 12 weeks in the programme) and hospitalisations (children referred for inpatient care at admission or at any point during the course of treatment).

\section{Data collection procedures and monitoring}

One of the five supervisors visited each health facility bimonthly to ensure protocol adherence and correct completion of patient records. There was no increase in clinical staff to manage the nutrition programme.

Data were collected using the national programme individual outpatient records (modified to include information on family MUAC-training). These forms were entered into an anonymised Access database. Data monitoring was conducted from completed archived patient records and included searching for each child's anonymous unique identifier in health centres in order to ensure that all children enrolled were in the database.

Socio-demographic information on the caretaker and child, mode of referral, distance to the health centre, caretaker MUAC-training prior to admission, Amoxicillin prescription and results of malaria rapid diagnostic test were collected at the first visit. The child's weight, MUAC, temperature, clinical symptoms and amount of RUTF ration were recorded at each weekly visit. Children's length was measured at admission and once a month thereafter. Weight was measured to the nearest $100 \mathrm{~g}$ with a Salter scale, and length was measured to the nearest $0.5 \mathrm{~cm}$ on a height board with the child in a supine position (or standing if taller than $85 \mathrm{~cm}$ ). MUAC was measured to the nearest $\mathrm{mm}$ with a MUAC bracelet demarcated in $1 \mathrm{~mm}$ increments. At each visit, supervisors ensured that scales were correctly calibrated and MUAC bracelets and height boards were in good condition.

\section{Data analysis}

Data analysis was performed using RStudio software (RStudio, Inc.).

WHZ, weight-for-age $Z$-score and height-for-age $Z$-score (HAZ) were calculated using WHO 2006 growth standards.

In order to evaluate adherence to the new OptiMA dosage tables, the difference between weekly RUTF ration provided to the child and the theoretical OptiMA ration as calculated by the new tables were described.

Children who were alive but erroneously classified as recovered (after a single MUAC measurement $\geq 125 \mathrm{~mm}$ ) or defaulter (prior to three consecutive weeks of absence) or non-respondent (before 12 weeks of participation without achieving discharge criteria), and who did not correspond to another category of exit, were reclassified as 'Alive, unconfirmed status'.

Programme outcomes were described overall and stratified by MUAC category at admission with their $95 \% \mathrm{CI}$ and considered at least as effective as the international SPHERE standards if the lower limit of the $95 \%$ CI was greater than or equal to the reference value. 


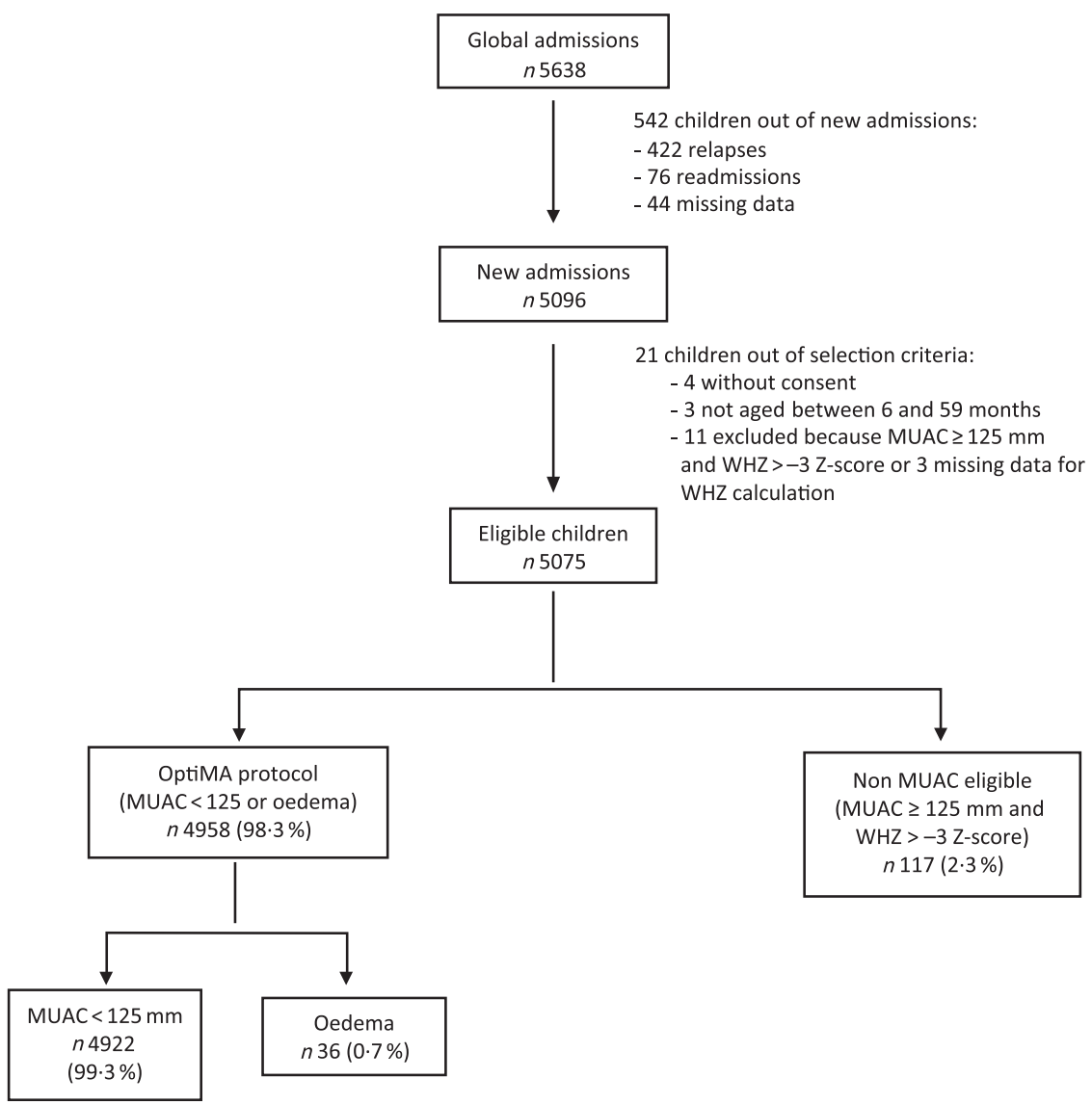

Fig. 1. Flow chart of children admitted under the Optimising treatment for acute MAlnutrition (OptiMA) protocol, Yako district, Burkina Faso, 2017. MUAC, mid upper arm circumference; $\mathrm{WHZ}$, weight-for-height $Z$-score.

Multivariable survival analysis to identify variables predictive of recovery was run using a shared frailty model ${ }^{(18)}$ (extension of the Cox proportional hazard model), with a random effect on health centres, and by assuming a Weibull distribution for the baseline hazard function. A univariate analysis by fitting a separate model for each covariate was done, variables having a $P$-value $\leq 0.2$ were entered into multivariate analysis. $P$-value $<0.05$ was considered as statistically significant in the final model.

To investigate the contribution of factors that explain the recovery (i.e. the proportion of recovered children explained by each variable of interest), adjusted attributable fraction (AF) of risks was estimated based on the final multivariate model. The AF for each factor was obtained by the difference between the predicted number of recovered children in a simulated dataset where exposure to each variable was removed and the real number of recovered children in OptiMA database ${ }^{(19)}$. The adjusted AF were estimated in relation to the recovery at the median length of stay in the programme. We computed $95 \%$ CI using bootstrapping ${ }^{(19)}$.

\section{Ethics}

The present study was approved by the Ethics Committee for Health Research (2016-6-067) and Technical Review Committee for Clinical Trial Authorizations (5003720165EC0000) of Burkina
Faso's Ministry of Health. The trial was registered on clinicaltrials.gov (NCT03027505). Caregivers gave written consent (signature or fingerprint) prior to enrollment for all children included in the study. All data were anonymised when entered into the database, and unique identification numbers were coded. A data safety monitoring board conducted an independent interim analysis, reviewed consolidated results and endorsed completion of the study.

\section{Results}

Of the 5638 children included in the nutrition programme from January to December 2017, 4958 were included for analysis (Fig. 1). Children omitted from analysis were those who relapsed, were readmitted, had missing data, did not meet OptiMA selection criteria or were not MUAC eligible (MUAC $\geq 125 \mathrm{~mm}$ and $\mathrm{WHZ}<-3$ ). Non-MUAC eligible children are described in online Supplementary file 1.

Baseline characteristics of children included under OptiMA are presented in Table 2. A majority were girls (58.4\%) and younger than 24 months $(82.2 \%)$. Overall, $0.7 \%$ of children were admitted with oedema, 15.9, 21.6 and $61.8 \%$ with MUAC $<115,115-119$ and 120-124 mm, respectively. Upon admission, $71.0 \%$ of children met both MUAC and WHZ definition for wasting (28.8\% with $\mathrm{WHZ}<-3$, and $42.2 \%$ with 
Table 2. Description of children included in Optimising treatment for acute MAlnutrition (OptiMA) protocol, Yako district, Burkina Faso, 2017 (Numbers and percentages; mean values and standard deviations)

\begin{tabular}{|c|c|c|c|c|c|c|c|c|}
\hline & \multicolumn{2}{|c|}{ Overall $(n$ 4958) } & \multicolumn{2}{|c|}{$\begin{array}{c}\text { MUAC }<115 \mathrm{~mm} \text { or } \\
\text { oedema }(n 824)\end{array}$} & \multicolumn{2}{|c|}{$\begin{array}{c}\text { MUAC 115-119 } \\
m m(n \text { 1070) }\end{array}$} & \multicolumn{2}{|c|}{$\begin{array}{c}\text { MUAC 120-124 } \\
\text { mm (n 3064) }\end{array}$} \\
\hline & $n$ & $\%$ & $n$ & $\%$ & $n$ & $\%$ & $n$ & $\%$ \\
\hline \multicolumn{9}{|l|}{ Child socio-demographics characteristics } \\
\hline \multicolumn{9}{|l|}{ Age (months) } \\
\hline Mean & \multicolumn{2}{|c|}{14.9} & \multicolumn{2}{|c|}{$12 \cdot 7$} & \multicolumn{2}{|c|}{$14 \cdot 5$} & \multicolumn{2}{|c|}{$15 \cdot 7$} \\
\hline SD & & & & & & & & \\
\hline Age category & & & & & & & & \\
\hline$<24$ months & 4077 & $82 \cdot 2$ & 728 & 88.3 & 901 & $84 \cdot 2$ & 2448 & 79.9 \\
\hline$\geq 24$ months & 881 & $17 \cdot 8$ & 96 & 11.7 & 169 & $15 \cdot 8$ & 616 & $20 \cdot 1$ \\
\hline Sex $(n$ 4941) & & & & & & & & \\
\hline Male & 2057 & 41.6 & 326 & 39.8 & 421 & 39.6 & 1310 & $42 \cdot 8$ \\
\hline Female & 2884 & $58 \cdot 4$ & 494 & $60 \cdot 2$ & 641 & $60 \cdot 4$ & 1749 & $57 \cdot 2$ \\
\hline Mode of referral ( $n$ 4845) & & & & & & & & \\
\hline Community agent & 1904 & $39 \cdot 3$ & 266 & 33.2 & 406 & $38 \cdot 7$ & 1232 & $41 \cdot 2$ \\
\hline Mothers screening & 628 & $12 \cdot 9$ & 154 & $19 \cdot 2$ & 158 & $15 \cdot 1$ & 316 & $10 \cdot 5$ \\
\hline Outpatient consultation & 2253 & 46.5 & 359 & 44.7 & 476 & $45 \cdot 3$ & 1418 & 47.4 \\
\hline Inpatient consultation & 60 & $1 \cdot 2$ & 23 & $2 \cdot 9$ & 10 & 0.9 & 27 & 0.9 \\
\hline Distance to the health centre ( $n$ 4703) & & & & & & & & \\
\hline$\geq 10 \mathrm{~km}$ & 447 & 9.5 & 113 & 14.4 & 122 & $12 \cdot 0$ & 212 & $7 \cdot 3$ \\
\hline$<10 \mathrm{~km}$ & 4256 & $90 \cdot 5$ & 670 & 85.6 & 894 & 88.0 & 2692 & 92.7 \\
\hline Mother's characteristics at admission & & & & & & & & \\
\hline Mother's status ( $n$ 4873) & & & & & & & & \\
\hline Alive & 4806 & 98.6 & 786 & 97.4 & 1038 & $98 \cdot 2$ & 2982 & $99 \cdot 1$ \\
\hline Dead & 67 & 1.4 & 21 & 2.6 & 19 & 1.8 & 27 & 0.9 \\
\hline No. of siblings ( $n$ 4697) & & & & & & & & \\
\hline 0 & 18 & 0.4 & 5 & 0.7 & 6 & 0.6 & 7 & 0.2 \\
\hline $1-4$ & 2711 & 57.7 & 433 & 55.9 & 595 & 59.4 & 1683 & $57 \cdot 6$ \\
\hline $4-12$ & 1968 & 41.9 & 336 & 43.4 & 400 & $40 \cdot 0$ & 1232 & $42 \cdot 2$ \\
\hline Breast-feeding ( $n$ 4512) & & & & & & & & \\
\hline Yes & 3820 & 84.7 & 652 & 87.7 & 808 & $84 \cdot 3$ & 2360 & 84.0 \\
\hline No & 692 & $15 \cdot 3$ & 91 & $12 \cdot 3$ & 151 & $15 \cdot 7$ & 450 & $16 \cdot 0$ \\
\hline Years of schooling ( $n$ 4442) & & & & & & & & \\
\hline No & 3941 & 88.7 & 672 & $90 \cdot 8$ & 855 & $89 \cdot 6$ & 2414 & $87 \cdot 8$ \\
\hline$\geq 1$ year & 501 & $11 \cdot 3$ & 68 & 9.2 & 99 & $10 \cdot 4$ & 334 & $12 \cdot 1$ \\
\hline MUAC training ( $n$ 4844) & & & & & & & & \\
\hline Yes & 3733 & $77 \cdot 1$ & 614 & $76 \cdot 2$ & 772 & $74 \cdot 2$ & 2347 & $78 \cdot 3$ \\
\hline No & 1111 & $22 \cdot 9$ & 192 & $23 \cdot 8$ & 268 & $25 \cdot 8$ & 651 & 21.7 \\
\hline Anthropometric characteristics & & & & & & & & \\
\hline MUAC at admission (mm) ( $n$ 4922) & & & & & & & & \\
\hline Mean & & & & & & & & \\
\hline SD & & & & & & & & \\
\hline WHZ ( $n$ 4777) & & & & & & & & \\
\hline Mean & & & & & & & & \\
\hline SD & & & & & & & & \\
\hline WHZ categories & & & & & & & & \\
\hline$<-3$ & 1377 & $28 \cdot 8$ & 423 & $56 \cdot 3$ & 357 & 34.6 & 597 & $19 \cdot 9$ \\
\hline-3 and -2 & 2017 & $42 \cdot 2$ & 239 & 31.8 & 450 & $43 \cdot 6$ & 1328 & $44 \cdot 3$ \\
\hline$\geq-2$ & 1383 & $29 \cdot 0$ & 89 & 11.9 & 224 & $21 \cdot 7$ & 1070 & $35 \cdot 7$ \\
\hline HAZ ( $n$ 4760) & & & & & & & & \\
\hline Mean & & & & & & & & \\
\hline SD & & & & & & & & \\
\hline HAZ categories & & & & & & & & \\
\hline$<-3$ & 848 & $17 \cdot 8$ & 205 & $27 \cdot 1$ & 217 & $21 \cdot 2$ & 426 & $14 \cdot 3$ \\
\hline-3 and -2 & 1150 & $24 \cdot 2$ & 216 & $28 \cdot 6$ & 253 & $24 \cdot 8$ & 681 & $22 \cdot 8$ \\
\hline$\geq-2$ & 2762 & 58.0 & 335 & 44.3 & 552 & 54.0 & 1875 & 62.9 \\
\hline WAZZ ( $n$ 4772) & & & & & & & & \\
\hline Mean & & & & & & & & \\
\hline SD & & & & & & & & \\
\hline WAZ categories & & & & & & & & \\
\hline$<-3$ & 1858 & 38.9 & 496 & $68 \cdot 7$ & 494 & $48 \cdot 1$ & 868 & $28 \cdot 7$ \\
\hline-3 and -2 & 2008 & $42 \cdot 1$ & 192 & $26 \cdot 6$ & 406 & 39.5 & 1410 & $46 \cdot 7$ \\
\hline$\geq-2$ & 906 & $19 \cdot 0$ & 34 & $4 \cdot 7$ & 128 & $12 \cdot 4$ & 744 & $24 \cdot 6$ \\
\hline Malaria data & & & & & & & & \\
\hline Malaria RDT ( $n$ 4293) & & & & & & & & \\
\hline Positive & 1103 & $25 \cdot 7$ & 192 & $26 \cdot 5$ & 275 & $29 \cdot 4$ & 636 & $24 \cdot 1$ \\
\hline Negative & 3190 & $74 \cdot 3$ & 532 & 73.5 & 659 & $70 \cdot 6$ & 1999 & $75 \cdot 9$ \\
\hline Received SMC ( $n$ 4907) & & & & & & & & \\
\hline Included out of SMC campaign & 3550 & $72 \cdot 3$ & 615 & $75 \cdot 4$ & 785 & 74.3 & 2150 & $70 \cdot 8$ \\
\hline SMC campaign eligible and received & 305 & $6 \cdot 2$ & 41 & $5 \cdot 0$ & 53 & $5 \cdot 0$ & 211 & $6 \cdot 9$ \\
\hline SMC campaign eligible and not received & 1052 & $21 \cdot 5$ & 160 & $19 \cdot 6$ & 218 & $20 \cdot 6$ & 674 & $22 \cdot 2$ \\
\hline
\end{tabular}

MUAC, mid upper arm circumference; WHZ, weight-for-height Z-score; HAZ, height-for-age Z-score; WAZ, weight-for-age Z-score; RDT, rapid diagnostic tests; SMC, seasonal malaria chemoprevention during campaign period from July to October 2017. 


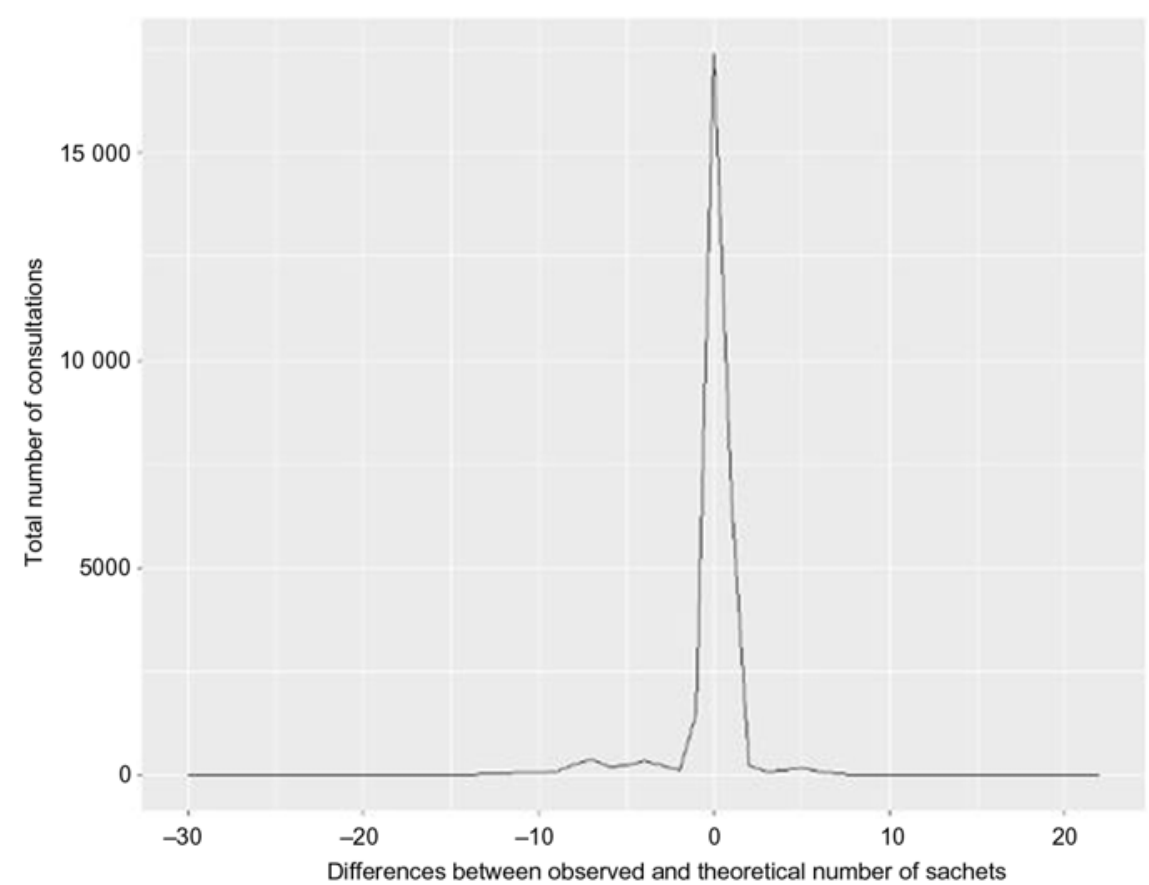

Fig. 2. Distribution of the differences by consultation between the number of ready-to-use therapeutic food sachets given to children and the theoretical dosage under Optimising treatment for acute MAlnutrition protocol, Yako district, Burkina Faso, 2017.

WHZ between -3 and -2), while $29.0 \%$ met the MUAC definition only. Stunting was present in $42.0 \%$ of children. The proportion and severity of wasting by WHZ, stunting and underweight were highest among children admitted with MUAC $<115 \mathrm{~mm}$. Most caretakers $(77 \cdot 1 \%)$ received MUAC training prior to child's admission.

Fig. 2 shows the difference by visit between the number of RUTF sachets given to children and the theoretical dosage per OptiMA protocol. The new dosage regimen was correctly implemented at all visits for $\mathbf{7 5 . 9} \%$ of children. For children who received a dosage error, the distribution shows that for most of the visits, the mistake was within two sachets. Mothers who notified health workers of a planned absence received 2 or 3 weeks of RUTF ration at one visit, which may explain the larger variations.

Overall recovery exceeded the SPHERE standard for both SAM and MAM programmes: $86.3 \%$ (95\% CI 85.4, 87.2) (Table 3 ). Recovery was lowest among children who were most malnourished at admission, with $70.4 \%$ (95\% CI 67.5, 73.5) of the children admitted with MUAC $<115 \mathrm{~mm}$ or oedema recovering. There were $243(4.9 \%)$ children who were alive at discharge but wrongly classified by nurses, with $122(50.0 \%)$ of these children erroneously classified as recovered with one MUAC measure $\geq 125 \mathrm{~mm}$ instead of two. Mortality rate was low $(0.4 \% ; 95 \%$ CI $0 \cdot 0,1.3)$, while defaults, non-response and transfers represented $4.7 \%$ (95\% CI 3.8, 5.6), 3.4\% (95\% CI $2 \cdot 5,4 \cdot 3)$ and $0 \cdot 2 \%(95 \%$ CI $0 \cdot 0,1 \cdot 1)$, respectively.

Table 4 shows programme outcomes stratified by MUAC and WHZ at admission. Children with both MUAC $<115 \mathrm{~mm}$ and WHZ $<-3$ had the lowest recovery $(64.3 \%$, 95\% CI 60.0, $68.9)$ and highest non-response $(12.5 \%, 95 \% \mathrm{CI} 8 \cdot 3,17 \cdot 1)$ and mortality $(1 \cdot 7 \%, 95 \%$ CI $0 \cdot 0,6 \cdot 2)$.
Home visits were conducted for 233 children classified as defaulters: 113 were alive and physically seen (average MUAC was 124, sD 7·1), thirty-eight were declared alive by the caretaker but not physically seen, four were deceased and seventy-eight could not be found. Among the seventy-seven defaulters with a MUAC $<115 \mathrm{~mm}$ at admission, the average number of visits before default was 4.7 and their last MUAC measure was $115 \cdot 1 \mathrm{~mm}$ on average (sD 8.2).

Of 170 non-responders, eighty-four (49.4\%) were admitted with MUAC $<115 \mathrm{~mm}$ and three $(1.8 \%)$ for oedema. The average length of stay and MUAC at last visit for all non-responders and those with MUAC $<115 \mathrm{~mm}$ or oedema at admission were $14 \cdot 6$ (sD 3.5) weeks, 120.4 (sD 5.6) mm and $15 \cdot 3$ (sD 3.8) weeks, 119.5 (sD 4.6) $\mathrm{mm}$, respectively.

Of the twenty-two deaths, $72.7 \%$ were younger than 24 months, $59 \cdot 1 \%$ were girls and $54.5 \%$ had a MUAC $<115$ or oedema at admission, while this group accounted for only $16.6 \%$ of all admissions. The average time from admission to death was 18.8 (sD 19.2) d, with six, eight and eight deaths occurring on the day of admission, 1-21 and 22-60 d after admission, respectively. Among these sixteen deaths that occurred after the day of admission, ten children had MUAC $<115 \mathrm{~mm}$, nine were girls and the median age was 14 months (interquartile range $7 \cdot 7,26 \cdot 2$ )

Among all recovered children and those admitted with MUAC $<115 \mathrm{~mm}$ or oedema, length of stay was 5.8 and 8.1 weeks, MUAC gain was 9.8 and $17.5 \mathrm{~mm}$, weight gain was 3.7 and $4.7 \mathrm{~g} / \mathrm{kg}$ per $\mathrm{d}$ and the amount of RUTF provided was 51.0 and 87.4 sachets, respectively (Table 5). The OptiMA dosage table provided a slightly lower than the anticipated average daily energetic intake to the most malnourished at $131 \mathrm{kcal} / \mathrm{kg}$ per d (548 kJ/kg per d). 
Table 3. Programme outcome globally and by mid upper arm circumference (MUAC) category at admission among children treated by Optimising treatment for acute MAlnutrition (OptiMA) protocol, Yako district, Burkina Faso, 2017

(Numbers and percentages; $95 \%$ confidence intervals; mean values and standard deviations)

\begin{tabular}{|c|c|c|c|c|c|c|c|c|c|c|c|c|c|c|}
\hline & \multirow{3}{*}{$\begin{array}{c}\text { National } \\
\text { standard (\%) }\end{array}$} & \multirow{3}{*}{$\begin{array}{l}\text { SPHERE } \\
\text { standard (\%) }\end{array}$} & \multirow{2}{*}{\multicolumn{3}{|c|}{ Overall ( $n$ 4958) }} & \multicolumn{9}{|c|}{ MUAC category at admission } \\
\hline & & & & & & \multicolumn{3}{|c|}{$\begin{array}{c}<115 \mathrm{~mm} \text { or oedema ( } n \\
824)\end{array}$} & \multicolumn{3}{|c|}{$115-119$ mm (n 1070) } & \multicolumn{3}{|c|}{ 120-124 mm (n 3064) } \\
\hline & & & $n$ & $\%$ & $95 \% \mathrm{Cl}$ & $n$ & $\%$ & $95 \% \mathrm{Cl}$ & $n$ & $\%$ & $95 \% \mathrm{Cl}$ & $n$ & $\%$ & $95 \% \mathrm{Cl}$ \\
\hline \multicolumn{15}{|l|}{ Exit status } \\
\hline Recovered & 75 & 75 & 4279 & $86 \cdot 3$ & $85 \cdot 4,87 \cdot 2$ & 580 & $70 \cdot 4$ & $67.5,73.5$ & 900 & $84 \cdot 1$ & $82 \cdot 1,86 \cdot 2$ & 2799 & 91.4 & $90 \cdot 4,92 \cdot 2$ \\
\hline Deceased & 3 & 10 & 22 & 0.4 & $0.0,1.3$ & 12 & 1.5 & $0.0,4.5$ & 6 & 0.6 & $0.0,2.6$ & 4 & 0.1 & $0.0,1.0$ \\
\hline Defaulted & 15 & 15 & 233 & 4.7 & $3.8,5 \cdot 6$ & 77 & $9 \cdot 3$ & $6 \cdot 4,12 \cdot 4$ & 59 & 5.5 & $3 \cdot 6,7 \cdot 6$ & 97 & $3 \cdot 2$ & $2 \cdot 2,4 \cdot 1$ \\
\hline Non-responders & & & 170 & $3 \cdot 4$ & $2 \cdot 5,4 \cdot 3$ & 87 & $10 \cdot 6$ & $7 \cdot 6,13 \cdot 6$ & 46 & $4 \cdot 3$ & $2 \cdot 3,6 \cdot 4$ & 37 & 1.2 & $0 \cdot 2,2 \cdot 1$ \\
\hline Transferred & & & 11 & 0.2 & $0.0,1 \cdot 1$ & 4 & 0.5 & $0.0,3.5$ & 1 & 0.1 & $0.0,2 \cdot 2$ & 6 & 0.2 & $0.0,1 \cdot 1$ \\
\hline Alive: unconfirmed status & & & 243 & 4.9 & $4 \cdot 0,5 \cdot 8$ & 64 & $7 \cdot 8$ & $4 \cdot 8,10 \cdot 8$ & 58 & 5.4 & $0.3,7.5$ & 121 & 3.9 & $3 \cdot 1,4 \cdot 9$ \\
\hline \multicolumn{15}{|l|}{ Hospitalisation } \\
\hline At least one hospitalisation & & & 680 & $13 \cdot 7$ & $12 \cdot 8,14 \cdot 7$ & 269 & $32 \cdot 6$ & $29 \cdot 5,36 \cdot 0$ & 186 & 17.4 & $15 \cdot 2,19 \cdot 7$ & 225 & $7 \cdot 3$ & $6 \cdot 4,8 \cdot 2$ \\
\hline Hospital mortality & & & 6 & 0.9 & $0.0,4.0$ & 3 & $1 \cdot 1$ & $0.0,6.5$ & 3 & 1.6 & $0.0,7.7$ & 0 & & \\
\hline \multicolumn{15}{|l|}{ Length of hospital stay (d) } \\
\hline Mean & & & & 8.4 & & & 9.2 & & & $8 \cdot 2$ & & & 7.5 & \\
\hline SD & & & & 5 & & & 5.7 & & & 4.5 & & & 4.2 & \\
\hline
\end{tabular}

Table 4. Programme outcomes among children treated with Optimising treatment for acute MAlnutrition (OptiMA) protocol stratified by mid upper arm circumference (MUAC) category and weight-for-height $Z$ score $(\mathrm{WHZ})^{*}$ at admission, Yako district, Burkina Faso, 2017

(Numbers and percentages; confidence intervals)

\begin{tabular}{|c|c|c|c|c|c|c|c|c|c|c|c|c|c|c|c|c|c|c|}
\hline & \multicolumn{3}{|c|}{$\begin{array}{c}\text { MUAC < 115; } \\
\text { WHZ <-3 ( } n \text { 423) }\end{array}$} & \multicolumn{3}{|c|}{$\begin{array}{c}\text { MUAC <115; } \\
\text { WHZ }-3 \text { and }-2 \text { ( } n \text { 239) } \\
\end{array}$} & \multicolumn{3}{|c|}{$\begin{array}{c}\text { MUAC }<115 \\
\text { WHZ } \geq-2(n 89) \\
\end{array}$} & \multicolumn{3}{|c|}{$\begin{array}{c}\text { MUAC 115-124; } \\
\text { WHZ < -3 (n 954) }\end{array}$} & \multicolumn{3}{|c|}{$\begin{array}{c}\text { MUAC 115-124; } \\
\text { WHZ -3 and }-2 \text { ( } n \text { 1778) } \\
\end{array}$} & \multicolumn{3}{|c|}{$\begin{array}{c}\text { MUAC 115-124; } \\
\text { WHZ } \geq-2 \text { (n 1294) }\end{array}$} \\
\hline & $n$ & $\%$ & $95 \% \mathrm{Cl}$ & $n$ & $\%$ & $95 \% \mathrm{Cl}$ & $n$ & $\%$ & $95 \% \mathrm{Cl}$ & $n$ & $\%$ & $95 \% \mathrm{Cl}$ & $n$ & $\%$ & $95 \% \mathrm{Cl}$ & $n$ & $\%$ & $95 \% \mathrm{Cl}$ \\
\hline Recovery & 272 & $64 \cdot 3$ & $60 \cdot 0,68 \cdot 9$ & 193 & $80 \cdot 8$ & $76 \cdot 6,85 \cdot 7$ & 69 & 77.5 & $69 \cdot 7,85 \cdot 5$ & 844 & 84.9 & $81 \cdot 2,89 \cdot 4$ & 1592 & 89.5 & $88 \cdot 2,90.9$ & 1176 & 90.9 & $89 \cdot 5,92 \cdot 3$ \\
\hline Deceased & 7 & $1 \cdot 7$ & $0 \cdot 0,6 \cdot 2$ & 2 & 0.8 & $0 \cdot 0,5 \cdot 7$ & 0 & & & 5 & 0.8 & $0 \cdot 0,5 \cdot 2$ & 2 & 0.1 & $0.0,1.4$ & 3 & 0.2 & $0 \cdot 0,1 \cdot 7$ \\
\hline Defaulted & 47 & $11 \cdot 1$ & $6 \cdot 8,15 \cdot 7$ & 13 & $5 \cdot 4$ & $1 \cdot 2,10 \cdot 4$ & 7 & 7.9 & $0 \cdot 0,15 \cdot 9$ & 39 & 5.4 & $1 \cdot 7,9 \cdot 8$ & 64 & $3 \cdot 6$ & $2 \cdot 3,4 \cdot 9$ & 41 & $3 \cdot 2$ & $1 \cdot 8,4 \cdot 6$ \\
\hline Non-respondent & 53 & $12 \cdot 5$ & $8 \cdot 3,17 \cdot 1$ & 17 & $7 \cdot 1$ & $2 \cdot 9,12 \cdot 1$ & 11 & $12 \cdot 4$ & $4 \cdot 5,20 \cdot 4$ & 18 & $5 \cdot 4$ & $1 \cdot 7,9 \cdot 8$ & 41 & $2 \cdot 3$ & $1 \cdot 0,3 \cdot 6$ & 21 & 1.6 & $2 \cdot 3,3 \cdot 1$ \\
\hline Transferred & 2 & 0.5 & $0.0,5 \cdot 1$ & 1 & 0.4 & $0 \cdot 0,5 \cdot 4$ & 0 & & & 3 & 0.4 & $0.0,4 \cdot 8$ & 1 & 0.1 & $0.0,1.4$ & 2 & 0.2 & $0.0,1 \cdot 6$ \\
\hline Unconfirmed status & 42 & 9.9 & $5 \cdot 7,14.5$ & 13 & $5 \cdot 4$ & $1 \cdot 2,10 \cdot 4$ & 2 & $2 \cdot 2$ & 0.08 .0 & 45 & 2.9 & $0 \cdot 0,7 \cdot 3$ & 78 & 4.4 & $3 \cdot 1,5 \cdot 7$ & 51 & 3.9 & $2 \cdot 5,5 \cdot 4$ \\
\hline At least one hospitalisation & 151 & $35 \cdot 7$ & $31 \cdot 2,40 \cdot 5$ & 67 & $28 \cdot 0$ & $22 \cdot 6,33 \cdot 9$ & 14 & $15 \cdot 7$ & $8 \cdot 9,23 \cdot 0$ & 121 & $12 \cdot 7$ & $10 \cdot 7,14 \cdot 8$ & 175 & $9 \cdot 8$ & $8 \cdot 5,11 \cdot 2$ & 89 & 6.9 & $5 \cdot 6,8 \cdot 3$ \\
\hline
\end{tabular}

* 181 children had missing data on 'WHZ category'. 
Table 5. Average length of stay, weight gain, mid upper arm circumference (MUAC) gain and average ready-to-use therapeutic food (RUTF) consumption in recovered children treated with the Optimising treatment for acute MAlnutrition (OptiMA) protocol, Yako district, Burkina Faso, 2017 (Mean values and standard deviations; medians and interquartile ranges (IQR))

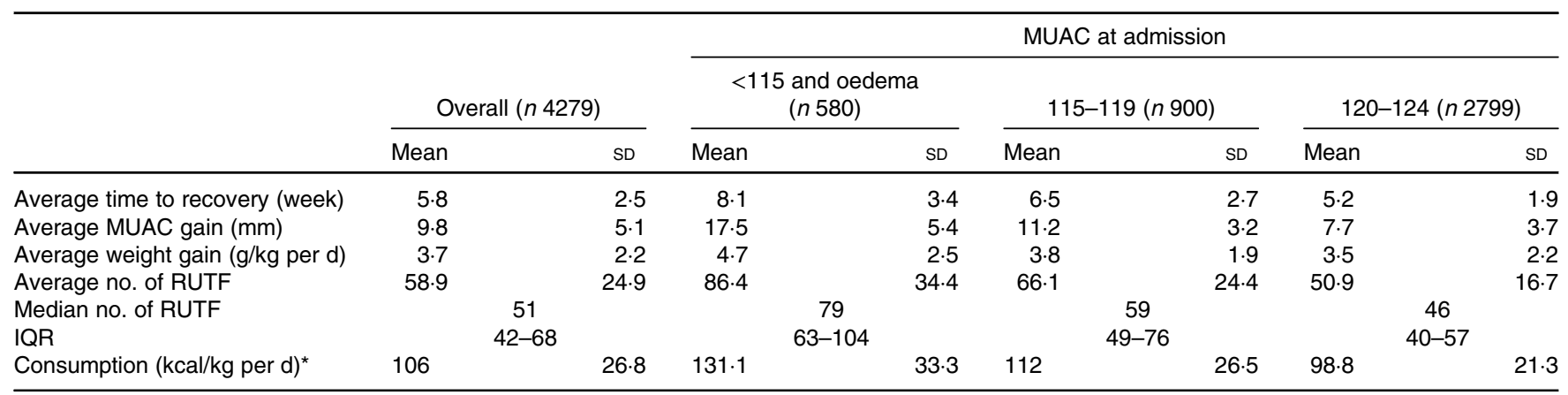

* To convert kcal to kJ, multiply by 4.184 .

Table 6. Factors associated and attributable fraction (AF) with nutritional recovery $(n 4163)$ among all children $(n 4958)$ included in Optimising treatment for acute MAlnutrition (OptiMA) protocol, Yako district, Burkina Faso, 2017*

(Numbers and percentages; hazard ratios (HR) and $95 \%$ confidence intervals)

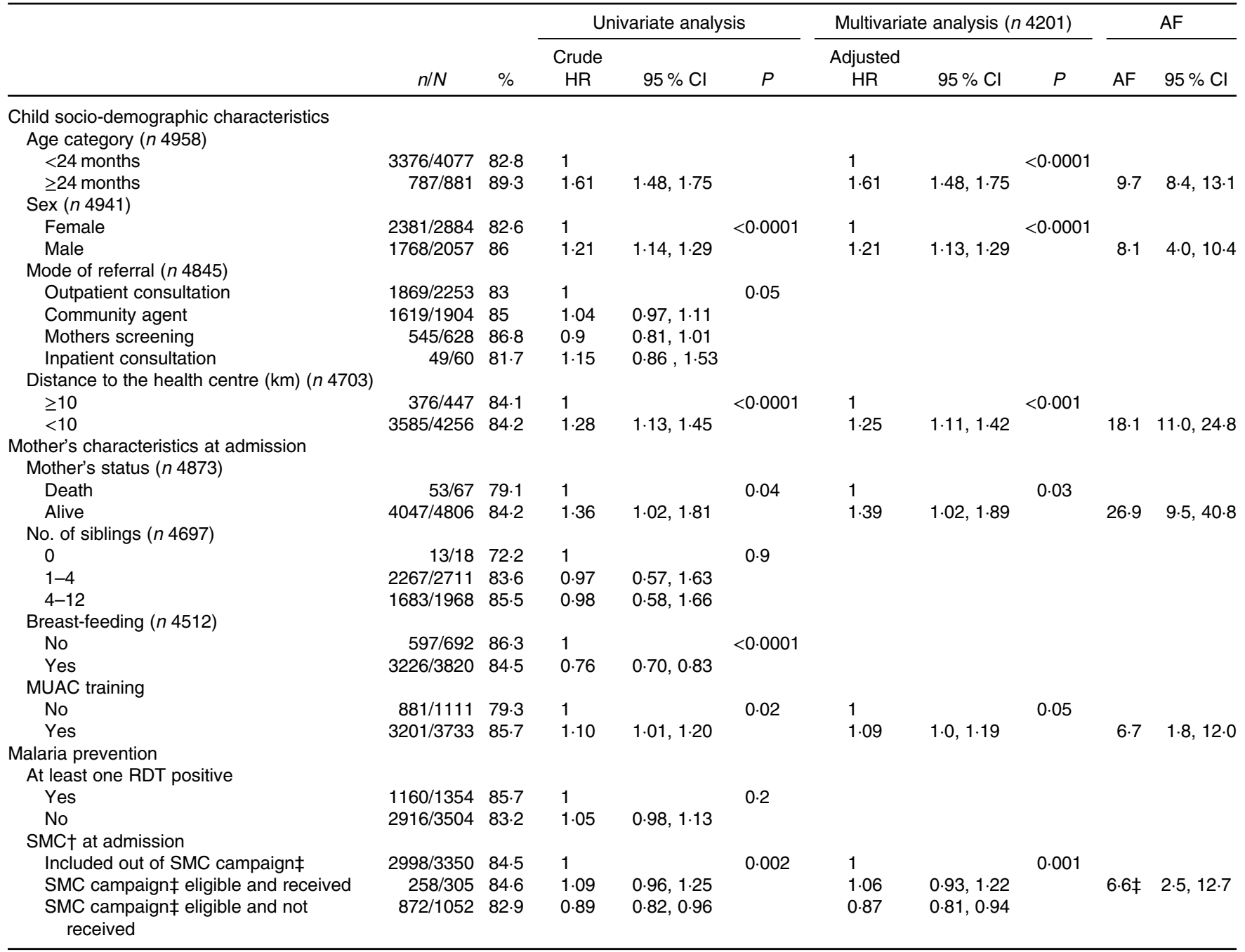

MUAC, mid upper arm circumference; RDT, rapid diagnostic tests; SMC, seasonal malaria chemoprevention.

* Shared frailty model with a random effect on health centres censoring at 12 weeks of inclusion in the programme.

† SMC campaign period from July to October 2017.

$\ddagger$ SMC at admission was combined in two categories 'included out of SMC campaign' and 'included during SMC campaign', the reference chosen for the AF calculation was 'included during SMC campaign'. 
UNICEF Burkina Faso plans an average of 140 sachets per SAM child treated. The comparable group in OptiMA is the 1778 children who met standard SAM admission criteria and whose average RUTF ration was reduced by nearly half at $72 \cdot 2$ sachets per child treated. The 3072 children treated under OptiMA who fit the current MAM case definition consumed an average of 54.3 RUTF sachets/child which is lower than the 60-90 ready-to-use supplementary food sachets/child typically planned for MAM programming.

Table 6 shows factors associated with recovery after controlling for age and sex: alive mother status (adjusted hazard ratio $1.39 ; 95 \%$ CI $1.02,1.89$ ), child of a caretaker who received MUAC training (adjusted hazard ratio 1.09; 95\% CI 1.01, 1.19) and who lived close to health centres (adjusted hazard ratio 1.25 ; $95 \%$ CI $1.11,1.42$ ) were more likely to recover. Conversely, children who did not receive seasonal malaria chemoprevention during July and October were less likely to recover $(0 \cdot 87 ; 95 \%$ CI 0.81, 0.94).

Globally, AF indicate that $57 \cdot 1 \%(95 \% \mathrm{CI} 46 \cdot 5,69 \cdot 6)$ of the recovery was explained by the six variables selected in the final model. The two variables that explain most of the recovery are: children whose mother is alive (AF 28.9\% (95\% CI 9.5, $40.8)$ and living within $10 \mathrm{~km}$ of the health centre $(18.1 \%$, $95 \%$ CI $11 \cdot 0,24 \cdot 8)$.

\section{Discussion}

This proof-of-concept trial evaluated a therapeutic nutrition protocol for children affected by acute malnutrition, defined as MUAC $<125 \mathrm{~mm}$ or oedema, and treated with one product (RUTF) at a gradually reduced dose based on a child's weight and MUAC status. The OptiMA protocol was implemented in a 'real-life' setting by Burkinabe Ministry of Health personnel in all fifty-four health centres of Yako District, with operational support from a national and international non-governmental consortium and an established UNICEF-supported RUTF supply chain. Nurses had minimal difficulty adapting to this new protocol as evidenced by excellent adherence to the new dosing table and application of the new admission criteria. Programme outcomes overall were satisfactory with substantial reduction in RUTF ration compared with standard SAM treatment. However, recovery and non-response rates were unsatisfactory in children admitted at MUAC $<115 \mathrm{~mm}$ or oedema.

Overall recovery and mortality rates reported here, $86 \cdot 3$ and $0.4 \%$ respectively, compare favourably to the $83 \%$ recovery and $4 \%$ mortality reported by a similar MUAC-based study in Sierra Leone, which had a more rapid reduction in RUTF dosage than OptiMA $^{(11)}$. A MUAC-based programme previously implemented in the same district of Burkina Faso and using MUAC $<120 \mathrm{~mm}$ for admissions reported $91.6 \%$ recovery and $1 \cdot 1 \%$ mortality, but the present study did not use a reduced RUTF regimen and had a less stringent discharge criterion of a single measure of MUAC $\geq 124 \mathrm{~mm}^{(6)}$.

Recovery in the category of children admitted at MUAC $<115 \mathrm{~mm}$ or oedema $(70.4 \%)$ was poorer than anticipated; this was largely attributable to high non-response $(10 \cdot 6 \%)$ and defaulter rates (9.3\%). Non-responders and defaulters did achieve an average MUAC of 119.5 and $115.1 \mathrm{~mm}$ at exit, respectively, while the average MUAC at admission in the MUAC $<115 \mathrm{~mm}$ group was $109.5 \mathrm{~mm}$. Mortality remained low at $1.5 \%$ and is similar to the mortality rate of $1.6 \%$ reported for children with MUAC $<115 \mathrm{~mm}$ at admission in the prior MUAC-based programme in this same district ${ }^{(6)}$. Elsewhere among MUAC-based programmes without RUTF reduction, recovery rates for this category have ranged from $57.4 \%$ in India, $63.4 \%$ in Malawi and $82 \%$ in Sudan ${ }^{(20-22)}$. In the MUAC-based programme with RUTF dose reduction in Sierra Leone recovery for this sub-group was $75.3 \%{ }^{(23)}$; however, nutritional oedema was more prevalent in Sierra Leone ( $2 \cdot 9 v \cdot 0 \cdot 7 \%$ in OptiMA). This range of reported recovery rates with and without RUTF dose reduction suggests that recovery for this category is challenging. Other important factors like underlying disease (HIV, tuberculosis, sickle cell disease) might have contributed to a low recovery rate.

Similarly, recovery rate was the lowest $(64.3 \%)$ for children admitted with MUAC $<115 \mathrm{~mm}$ and WHZ $<-3(8.8 \%$ of all admissions) and is again driven primarily by a high nonresponse rate at $12.5 \%$. Stunting was also highly prevalent in this group, reaching $57.9 \%$. Previous studies described a correlation between shorter length and treatment non-response rate, suggesting that severely wasted and stunted children may require other interventions over a longer time period to improve growth trajectory ${ }^{(7,24)}$.

The sub-optimal outcomes for the small proportion of severely wasted and stunted children should be interpreted in light of overall programme performance. Under OptiMA, only $16 \%$ of children treated were admitted with MUAC $<115 \mathrm{~mm}$ or oedema, while $84 \%$ were admitted with MUAC between 115 and $124 \mathrm{~mm}$, whereas the proportion of children in each category in the Sierra Leone study was 30 and $70 \%{ }^{(11)}$. This might suggest that the OptiMA programme achieved good coverage and caught most children early in the wasting process. Thus, those children who exhibit severe wasting and stunting, in spite of apparently good access to treatment, may represent either a history of low birth weight or malnutrition secondary to chronic illness, both of which lower expectations for treatment outcomes.

Current SAM programmes typically plan RUTF consumption between 120 and 150 sachets per child treated, while MAM programmes plan between sixty and ninety sachets of ready-to-use supplementary food per child ${ }^{(25,26)}$. However, comparisons of average rations must consider variations in discharge criteria. Under OptiMA, discharge was stringent: two consecutives measures of MUAC $\geq 125 \mathrm{~mm}$. In this light, the overall RUTF consumption of $60 \cdot 8$ sachets average per child per course of treatment is highly encouraging. However, further study will be needed to determine if increasing RUTF dosage for children admitted with MUAC $<115 \mathrm{~mm}$ will improve recovery and nonresponse even if other factors appear to influence recovery rate as shown in the multivariate analysis.

Female sex bias in MUAC-based nutrition programmes has been documented in previous studies ${ }^{(11,27)}$, similar to what the present study observed with $58.4 \%$ of girls at admission. Isanaka et al. also clearly show that WHZ score preferentially 
selects males ${ }^{(27)}$. Thus combining anthropometric criteria, MUAC and WHZ serve to maintain sex balance in nutrition programmes. Using MUAC as the single anthropometric criterion for programme admission may require improving MUAC sensitivity for boys and specificity for girls.

The multivariable survival model highlighted that recovery was positively associated with male children older than $24 \mathrm{~m}$, living within $10 \mathrm{~km}$ of the health facility, whose mother was alive and had been trained to use MUAC. Recovery was negatively associated with children who had not received seasonal malaria chemoprevention between July and October. Most of these results have already been found in other studies. It is well known that female and younger children present lower $\operatorname{MUAC}^{(28)}$. As for distance to the health facility, a study from Ethiopia showed that SAM children living within $25 \mathrm{~min}$ of the facility were 1.53 times more likely to recover ${ }^{(29)}$. It has also been shown that mothers screening their children by MUAC can lead to earlier initiation of care ${ }^{(12)}$. The low proportion of self-referral by mothers (12.9\%) is likely due to an underestimation because many mothers sought confirmation from a Community Health Worker (CHW) after using MUAC at home and then reported being referred by a $\mathrm{CHW}$ at admission. To the best of our knowledge, it is the first time that a positive association is demonstrated between training mother to use MUAC bracelet and recovery, likely due to a better care-seeking behaviour resulting from such trainings. The negative association between children who did not receive CPS should be viewed with caution because the reason why these children did not receive the seasonal malaria chemoprevention was unknown and we cannot exclude a confusion bias or even reverse causality. Of these six variables associated with recovery, AF calculation highlighted, unsurprisingly, that recovery was primarily driven by maternal vital status and living within $10 \mathrm{~km}$ of the health facility.

The main limitation in the present study is the absence of a comparator group. In order to determine how MUAC-based integrated SAM and MAM protocols perform compared with current protocols that rely on MUAC and WHZ criteria, additional randomised trials, in multiple contexts, are necessary. Another limitation is that we did not collect data on possible RUTF sharing. But, it was previously shown in a study on MAM treatment in the same location that nearly two-thirds of children consumed all of the prescribed LNS ration themselves ${ }^{(30)}$.

Lastly, this analysis relied on routinely collected programme data. Extensive data monitoring was carried out throughout the study to ensure that all children found in health centre registers were retrieved in the OptiMA database. To our knowledge, such monitoring is rarely performed in programmatic studies. The strength of the present study is that it shows it is possible to conduct robust data monitoring in a 'real-life' programme to generate a high-quality individualised database. However, it was not without challenge. The reclassification of children erroneously categorised at exit-influenced OptiMA's recovery rate downwards. Adherence to routine amoxicillin prescription for children with MUAC $<120 \mathrm{~mm}$ or oedema as specified in the protocol was uneven and thus precluded any analysis of correlation between this prescription and recovery.

\section{Conclusion}

A simplified, combined SAM and MAM protocol based on early detection through household MUAC screenings, treatment with one product determined by the presence of oedema and/or MUAC $<125 \mathrm{~mm}$, and progressive RUTF dose reduction had programme outcomes that exceeded national Burkina Faso and international SPHERE standards. This early detection and treatment initiation with progressively reduced RUTF ration shows promise and warrants further operational pilots in different contexts. However, randomised control trials are needed to compare the strategy to current national protocols ${ }^{(31)}$. Because of low recovery among children admitted with MUAC $<115 \mathrm{~mm}$, further study is also needed to determine whether increasing RUTF dosage for the most severely malnourished will improve outcomes.

\section{Acknowledgements}

The authors are indebted to the women and children who participated in the study. The authors wish to particularly acknowledge the ALIMA operational team on the ground who ran the study on a day-to-day basis.

The present study was developed as part of the Clinical and Operational Research Alliance (CORAL) research platform aimed at developing high-quality innovative and transformative research programmes within a partnership between scientists from the Inserm (Institut National de la Santé et la Recherche Médicale, Bordeaux and Abidjan) and the humanitarian organisation ALIMA (Alliance for International Medical Action), primarily on improvement of maternal and child health outcomes. The scientific policy of the CORAL partnership alongside with the supervision of research projects and dissemination of results is defined by a board of directors consisting of senior representatives from both ALIMA and Inserm: Renaud Becquet (Inserm Bordeaux, methodological co-chair), Susan Shepherd (ALIMA, medical co-chair), Augustin Augier (ALIMA), Solenne Barbe (ALIMA), Marie Jaspard (ALIMA \& Inserm Abidjan), Claire Levy-Marchal (ALIMA) and Xavier Anglaret (Inserm Bordeaux \& Abidjan). The CORAL partnership meets annually with an external scientific advisory group to review projects and strategic orientation.

The authors acknowledge the members of the data safety monitoring board (DSMB) for the OptiMA trial: Rebecca Grais (Epicentre, Médecins sans Frontières, Paris, France), Charles Yameogo (Institut de recherche en sciences de la santé, Ouagadougou, Burkina Faso) and Katia Castetbon (Ecole de santé publique, Université Libre de Bruxelles, Bruxelles, Belgium).

The study was funded by the European Union's humanitarian aid funds; the US Agency for International Development's Office and the Médecins Sans Frontières Foundation. The funders had no role in study design, data collection and analysis, decision to publish or preparation of the manuscript.

This document covers humanitarian aid activities implemented with the financial assistance of the European Union. The views expressed herein should not be taken, in any way, to reflect the official opinion of the European Union, and the 
European Commission is not responsible for any use that can be made of the information it contains.

S. S. developed the study concept. S. S., K. P., S. K., M. D. and R. B. wrote the study protocol and designed the study; M. D., M. I., K. I. and V. M. coordinated the study teams; M. D., M. I., K. I., V. M. and R. B. organised and supervised data collection; M. D., K. P., S. S. and R. B. developed the statistical analysis strategy; M. D. performed the statistical analysis; M. D. and K. P. wrote the first draft of the manuscript; S. S. and R. B. were primarily responsible for the final content. All authors actively participated in the interpretation of results, critically reviewed the first draft and had a substantial writing contribution to the development of the final manuscript.

K. P. serves on the Social Purposes Advisory Commission of Nutriset, a main producer of lipid-based nutrient supplement products. No other interests are declared.

\section{Supplementary material}

For supplementary material referred to in this article, please visit https://doi.org/10.1017/S0007114519003258

\section{References}

1. Black RE, Victora CG, Walker SP, et al. (2013) Maternal and child undernutrition and overweight in low-income and middle-income countries. Lancet 382, 427-451.

2. United Nations Children's Fund (UNICEF), World Health Organization, International Bank for Reconstruction and Development/The World Bank (2019). Levels and trends in child malnutrition: key findings of the 2019 Edition of the Joint Child Malnutrition Estimates, Licence: CC BY-NC-SA 3.0 IGO. Geneva: World Health Organization.

3. Osgood-Zimmerman A, Millear AI, Stubbs RW, et al. (2018) Mapping child growth failure in Africa between 2000 and 2015. Nature 555, 41-47.

4. Rogers E, Myatt M, Woodhead S, et al. (2015) Coverage of community-based management of severe acute malnutrition programmes in twenty-one countries, 2012-2013. PLOS ONE 10, e 0128666

5. Briend A, Mwangome MK \& Berkley JA (2019) Using midupper arm circumference to detect high-risk malnourished patients in need of treatment. In Handbook of Famine, Starvation, and Nutrient Deprivation, pp. 705-721 [V Preedy and V Patel, editors]. Cham: Springer.

6. Isanaka S, Hanson KE, Frison S, et al. (2018) MUAC as the sole discharge criterion from community-based management of severe acute malnutrition in Burkina Faso. Matern Child Nutr 15, e12688.

7. Binns P, Dale N, Hoq M, et al. (2015) Relationship between mid upper arm circumference and weight changes in children aged 6-59 months. Arch Public Health 73, 54.

8. Ackatia-Armah RS, McDonald CM, Doumbia S, et al. (2015) Malian children with moderate acute malnutrition who are treated with lipid-based dietary supplements have greater weight gains and recovery rates than those treated with locally produced cereal-legume products: a community-based, cluster-randomized trial. Am J Clin Nutr 101, 632-645.

9. No Wasted Lives (2019) The State of Acute Malnutrition. https://acutemalnutrition.org/en/countries (accessed March 2019).
10. World Food Programme (WFP) (2017) Global burden of acute malnutrition and WFP's response. https://analytics.wfp.org/ views/MAMdashboard/MAMDashboard?\%3Adisplay_count= no\&\%3Aembed=y\&\%3AshowAppBanner=false\&\%3AshowViz Home $=$ no\&iframeSizedToWindow $=$ true (accessed March 2019).

11. Maust A, Koroma AS, Abla C, et al. (2015) Severe and moderate acute malnutrition can be successfully managed with an integrated protocol in Sierra Leone. J Nutr 145, 2604-2609.

12. Alé F, Phelan KP, Issa H, et al. (2016) Mothers screening for malnutrition by mid-upper arm circumference is non-inferior to community health workers: results from a large-scale pragmatic trial in rural Niger. Arch Public Health 74, 38.

13. Isanaka S, Menzies NA, Sayyad J, et al. (2017) Cost analysis of the treatment of severe acute malnutrition in West Africa. Matern Child Nutr 13, e12398.

14. Isanaka S, Barnhart DA, McDonald CM, et al. (2019) Costeffectiveness of community-based screening and treatment of moderate acute malnutrition in Mali. BMJ Glob Health 4, e001227.

15. Burkina Faso Ministry of Health (2014) Protocol for the integrated management of acute malnutrition [in French].

16. Sphere Association (2011) The Sphere Handbook: Humanitarian Charter and Minimum Standards in Humanitarian Response, 3rd ed. Geneva, Switzerland. www.spherestandards.org/handbook (accessed March 2019).

17. Burkina Faso Ministry of Health (2016) 2016 National nutritional survey: Final report [in French].

18. Rondeau V, Marzroui Y \& Gonzalez JR (2012) Frailtypack: an R package for the analysis of correlated survival data with frailty models using penalized likelihood estimation or parametrical estimation. J Stat Softw 47, 28.

19. Arikawa S, Rollins N, Jourdain G, et al. (2018) Contribution of maternal antiretroviral therapy and breastfeeding to 24-month survival in human immunodeficiency virus-exposed uninfected children: an individual pooled analysis of African and Asian studies. Clin Infect Dis 66, 1668-1677.

20. Burza S, Mahajan R, Marino E, et al. (2015) Community-based management of severe acute malnutrition in India: new evidence from Bihar. Am J Clin Nutr 101, 847-859.

21. Binns PJ, Dale NM, Banda T, et al. (2016) Safety and practicability of using mid-upper arm circumference as a discharge criterion in community based management of severe acute malnutrition in children aged 6 to 59 months programmes. Arch Public Health 74, 24.

22. Dale NM, Myatt M, Prudhon C, et al. (2013) Using mid-upper arm circumference to end treatment of severe acute malnutrition leads to higher weight gains in the most malnourished children. PLOS ONE $\mathbf{8}$, e55404.

23. Manary M \& Phelan K (2019) Personal communication, March 5.

24. Khara T, Mwangome M, Ngari M, et al. (2018) Children concurrently wasted and stunted: a meta-analysis of prevalence data of children 6-59 months from 84 countries. Matern Child Nutr 14, e12516.

25. United Nations International Children's Emergency Fund (UNICEF) (2013) Ready-to-use therapeutic food for children with severe acute malnutrition. Position Paper No. 1. https://www.unicef.org/media/files/Position_Paper_Readyto-use_therapeutic_food_for_children_with_severe_acute_ malnutrition_June_2013.pdf (accessed March 2019).

26. Global Nutrition Cluster MAM Task Force (2017) Moderate acute malnutrition: a decision tool for emergencies. https:// reliefweb.int/sites/reliefweb.int/files/resources/DECISIONTOOL-FOR-MAM_w-exceptional-cicumstances_-May-2017update-final1.pdf (accessed March 2019).

27. Isanaka S, Guesdon B, Labar AS, et al. (2015) Comparison of clinical characteristics and treatment outcomes of children 
selected for treatment of severe acute malnutrition using mid upper arm circumference and/or weight-for-height $Z$-score. PLOS ONE 10, e0137606.

28. Grellety E, Krause LK, Shams Eldin M, et al. (2015) Comparison of weight-for-height and mid-upper arm circumference (MUAC) in a therapeutic feeding programme in South Sudan: is MUAC alone a sufficient criterion for admission of children at high risk of mortality? Public Health Nutr 18, 2575-2581.

29. Kabalo MY \& Seifu CN (2017)Treatment outcomes of severe acute malnutrition in children treated within Outpatient Therapeutic Program (OTP) at Wolaita Zone, Southern
Ethiopia: retrospective cross-sectional study. J Health Popul Nutr 36, 7.

30. Iuel-Brockdorf AS, Draebel TA, Ritz C, et al. (2016) Evaluation of the acceptability of improved supplementary foods for the treatment of moderate acute malnutrition in Burkina Faso using a mixed method approach. Appetite 99, 34-45.

31. Clinical Trials Registry (2018) ClinicalTrials.gov Identifier: NCT03751475. Optimizing Acute Malnutrition Management in Children Aged 6 to 59 Months in Democratic Republic of Congo (OptiMA-DRC). https://clinicaltrials.gov/ct2/show/ NCT03751475 